\title{
New Star Observations with NuSTAR: Flares from Young Stellar Objects in the $\rho$ Ophiuchi Cloud Complex in Hard X-Rays
}

\author{
Juliana T. Vievering ${ }^{1}$ (D), Lindsay Glesener ${ }^{1}$ (D), Brian W. Grefenstette ${ }^{2}$ (D), and David M. Smith ${ }^{3}$ (D) \\ ${ }^{1}$ School of Physics \& Astronomy, University of Minnesota, Twin Cities, Minneapolis, MN 55455, USA; vieve004@umn.edu \\ ${ }^{2}$ Cahill Center for Astronomy and Astrophysics, California Institute of Technology, Pasadena, CA 91125, USA \\ ${ }^{3}$ Santa Cruz Institute for Particle Physics and Department of Physics, University of California, Santa Cruz, CA 95064, USA \\ Received 2018 October 25; revised 2019 June 7; accepted 2019 June 26; published 2019 September 4
}

\begin{abstract}
We study the structure and dynamics of extreme flaring events on young stellar objects (YSOs) observed in hard X-rays by the Nuclear Spectroscopic Telescope Array (NuSTAR). During 2015 and 2016, NuSTAR made three observations of the star-forming region $\rho$ Ophiuchi, each with an exposure $\sim 50 \mathrm{ks}$. NuSTAR offers unprecedented sensitivity above $\sim 7 \mathrm{keV}$, making this data set the first of its kind. Through improved coverage of hard X-rays, it is finally possible to directly measure the high-energy thermal continuum for hot plasmas and to sensitively search for evidence of nonthermal emission from YSO flares. During these observations, multiple flares were observed, and spectral and timing analyses were performed on three of the brightest flares. By fitting an optically thin thermal plasma model to each of these events, we found flare plasma heated to high temperatures $(\sim 40-80 \mathrm{MK})$ and determined that these events are $\sim 1000$ times brighter than the brightest flares observed on the Sun. Two of the studied flares showed excess emission at $6.4 \mathrm{keV}$, and this excess may be attributable to iron fluorescence in the circumstellar disk. No clear evidence for a nonthermal component was observed, but upper limits on nonthermal emission allow for enough nonthermal energy to account for the estimated thermal energy in the flare on protostar IRS 43, which is consistent with the standard model for solar and stellar flares.
\end{abstract}

Unified Astronomy Thesaurus concepts: Star forming regions (1565); Stellar flares (1603); Stellar x-ray flares (1637); Pre-main sequence stars (1290); Young stellar objects (1834); Stellar activity (1580)

\section{Introduction}

Observed flares on distant stars are typically assumed to be similar to flares on our own Sun. Standard models for solar flares theorize that these energetic events are driven by magnetic reconnection, and during this process, a significant portion of the dissipated magnetic energy $(\sim 40 \%)$ is converted into kinetic energy of particles (e.g., Aschwanden et al. 2016). These accelerated particles then travel along magnetic field lines, producing nonthermal bremsstrahlung emission through interactions with dense chromospheric plasma (Brown 1971) and heating the ambient plasma to high temperatures $(>10 \mathrm{MK})$. This heated plasma then expands into the flare loop in a process called chromospheric evaporation and produces thermal bremsstrahlung emission. In this model, both thermal and nonthermal processes result in emission of X-rays, and thus, spectroscopic measurements in the X-ray regime are key to understanding the nature of energy release and transfer in flares.

When studying stellar flares, young stellar objects (YSOs) are particularly interesting targets as their heightened magnetic activity leads to extreme flaring events. The term YSO covers the early stages of a star's life, from infalling protostar $\left(\sim 10^{4} \mathrm{yr}\right)$ to weak-lined T Tauri stars $\left(\sim 10^{7} \mathrm{yr}\right)$. From early infrared-millimeter observations of YSOs, Lada \& Wilking (1984) developed an evolutionary classification system (Class I through Class III) based on characteristics of spectral energy distributions in this waveband, with higher class numbers corresponding to more evolved YSOs (Wilking \& Lada 1983).

Along with hosting extreme flaring events, YSOs also prove to be interesting sources due to the presence of circumstellar disks, which allows for the possibility of different flare loop configurations, such as photosphere-disk and disk-disk, in addition to the photospheric footpoints for flares on solar-type and M dwarf stars (Feigelson \& Montmerle 1999). Though the dense circumstellar material associated with YSOs strongly attenuates emission in certain wavebands, including the optical, higher-energy emission in the X-ray regime can be transmitted and measured by X-ray observatories.

Observations of intense X-ray flares on YSOs can additionally provide an opportunity to investigate the impact of high-energy radiation on the surrounding environment. One major question regarding YSOs is whether their flaring activity has an impact on planet formation. If enough high-energy $\mathrm{X}$-ray emission penetrates the protoplanetary disk, it is possible that the disk material could become sufficiently ionized to lead to magnetorotational instabilities (MRIs; Feigelson 2010).

YSOs have previously been observed in the X-ray regime by observatories such as Chandra and XMM-Newton (see Imanishi et al. 2001; Pillitteri et al. 2010). Surveys of the nearby starforming region $\rho$ Ophiuchi ( $\sim 120 \mathrm{pc}$; Loinard et al. 2008) by both observatories have detected many YSO flares and found through spectral analyses, that Class I sources are associated with hotter temperatures and larger absorption columns than their older counterparts. Additionally, these surveys have led to the discovery of interesting spectral features, such as the first detected $6.4 \mathrm{keV}$ line from a Class I source, which has been attributed to iron fluorescence (Imanishi et al. 2001). However, due to limited sensitivity at higher X-ray energies, these studies do not measure or place constraints on nonthermal emission, which is essential for understanding the energy transfer from nonthermal electrons to heating of plasma.

The Nuclear Spectroscopic Telescope Array $(N u S T A R)$ is the first satellite to use focusing optics in the hard X-ray regime and overtakes the effective are of previous X-ray imaging observatories above $\sim 6-7 \mathrm{keV}$. With improved 
Table 1

NuSTAR Observations of $\rho$ Ophiuchi

\begin{tabular}{lccc}
\hline \hline Sequence ID & Start Date and Time (UT) & End Date and Time (UT) & Exposure (ks) \\
\hline 30102028002 & 2015-May 10 10:31:07 & 2015-May-11 14:51:07 & 55 \\
30102028004 & 2015-Aug-25 18:56:08 & 2015-Aug-26 23:36:08 & 51 \\
30102028006 & 2016-Apr-29 09:36:08 & 2016-Apr-30 11:11:08 & 46 \\
\hline
\end{tabular}

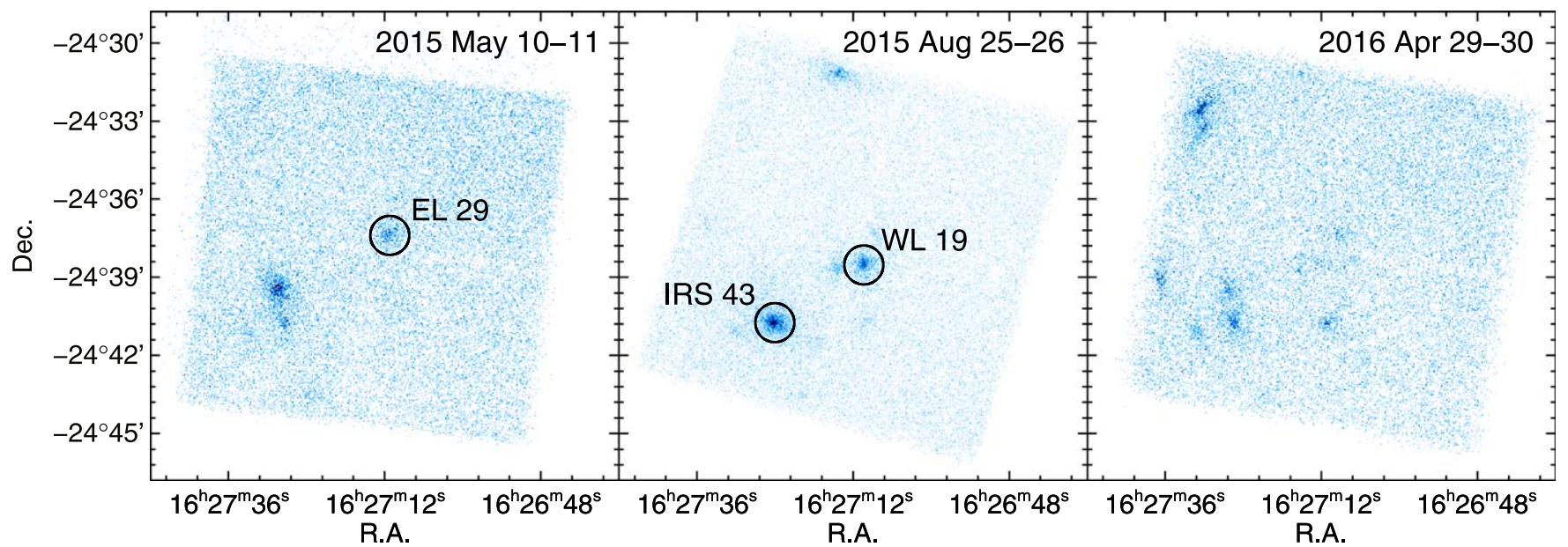

Figure 1. Images from FPMA show the time integrated NuSTAR observations of $\rho$ Ophiuchi over the whole FOV and the full energy range of 3-79 keV. Images are not background subtracted.

coverage of higher-energy X-rays, it is possible to search for evidence of nonthermal emission, to directly measure thermal continuum of hot plasmas, and to investigate the impact of high-energy radiation on circumstellar disks. NUSTAR performed the first focused hard X-ray $(\gtrsim 10 \mathrm{keV})$ observations of YSOs during 2015 and 2016 through three $\sim 50 \mathrm{ks}$ exposures of $\rho$ Ophiuchi. Over the course of these observations, NUSTAR observed multiple X-ray flares from YSOs, and the brightest of these events are analyzed here. Section 2 introduces the observations and outlines the process for data reduction. In Section 3, the analysis of flare spectra is described, and the corresponding results are presented. Section 4 offers a discussion of the results, and Section 5 provides a summary of our study.

\section{Observations and Data Processing}

The star-forming region $\rho$ Ophiuchi was observed by NuSTAR over three $\sim 50 \mathrm{ks}$ exposures during 2015 and 2016 as part of NuSTAR's Guest Observer Program (see Table 1).The NuSTAR science instrument is composed of two grazing incidence telescopes that are optimized over the energy range of 3-79 keV (Harrison et al. 2013). Each focal plane module, focal plane module (FPMA) and focal plane module B (FPMB), contains a $2 \times 2$ array of pixellated cadmium zinc telluride detectors, leading to small crosshair gaps in the image. Data from FPMA/FPMB were processed using the NuSTAR data analysis software (NuSTARDAS ${ }^{4}$ v1.6.0).

The flares analyzed here were selected by eye from full fieldof-view (FOV) images integrated over the entire observation period (see Figure 1). Multiple distinct flares were observed during the three observing intervals, and three of these flares have been analyzed in depth (see Figure 2 for corresponding

\footnotetext{
4 https://heasarc.gsfc.nasa.gov/docs/nustar/analysis/nustar_swguide.pdf
}
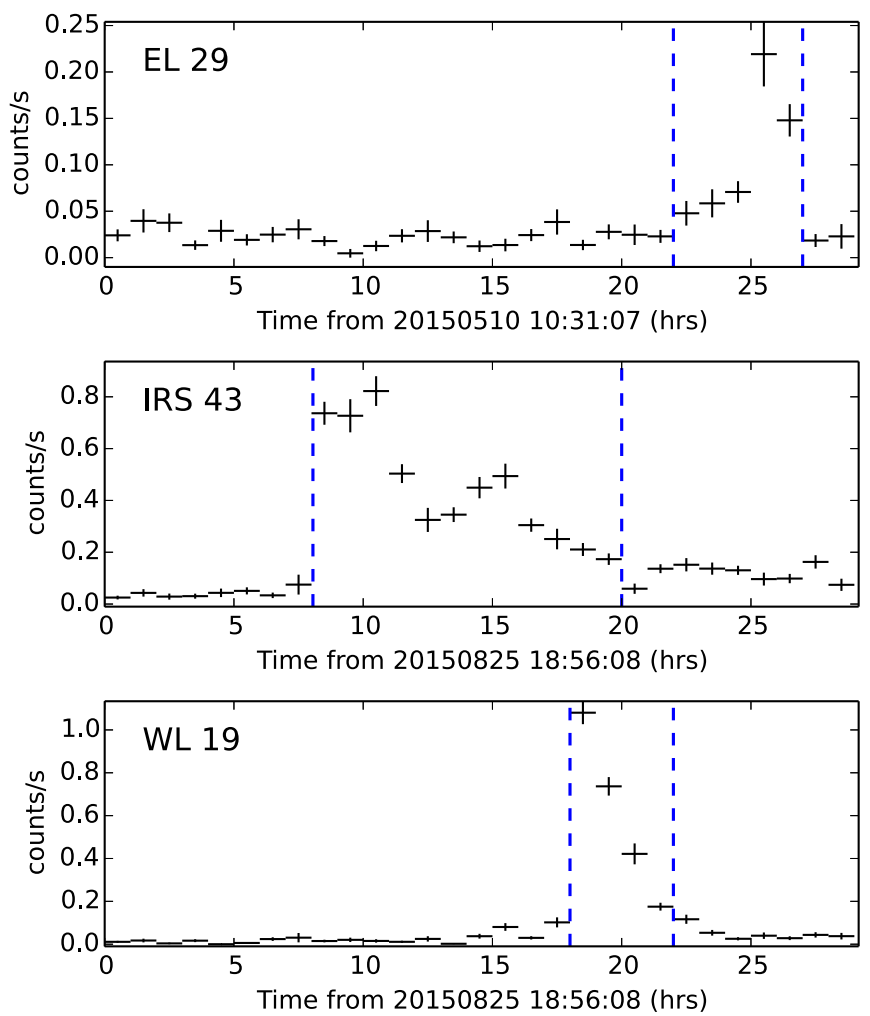

Figure 2. Light curves (binned by hour) of three YSO flares during the first two NuSTAR observations of $\rho$ Ophiuchi with combined data from FPMA and FPMB over the full energy range of 3-79 keV. Dashed lines indicate the time interval selected for the flare spectral analysis.

light curves). Sources were identified by comparing the flare location with catalogs from previous surveys of $\rho$ Ophiuchi (Imanishi et al. 2001; Pillitteri et al. 2010). During the first 
observation, Class I protostar Elias 29 (hereafter EL 29; Elias 1978) produced a flare that lasted $\sim 5 \mathrm{hr}$. Two large flares occurred during the second observation: one from Class I protostar IRS 43 (Wilking et al. 1989) and one from Class III source WL 19 (Wilking \& Lada 1983). These flares lasted $\sim 12.6$ and $\sim 4.2 \mathrm{hr}$, respectively. In future analyses, we will both examine the bright additional flares visible in Figure 1 and search for other potential sources near the sensitivity limits of NUSTAR.

\section{Analysis}

\subsection{Background Estimation}

The relatively low background of NUSTAR $\left(\sim 10^{-3}\right.$ counts $\mathrm{s}^{-1}$ at $10-30 \mathrm{keV}$ ) within the half-power diameter includes focused cosmic X-ray background (CXB), unfocused CXB through the open light path, environmental neutrons, and instrument background (e.g., fluorescence lines). The backgrounds for the sources studied here were simulated through use of the nuskybgd suite of IDL routines (Wik et al. 2014). In this method, a source-free region of the FOV is selected-in our case, an annulus around each flaring YSO; each background component has a known spectral shape, and a fit of the normalizations of these components is performed based on the selected background region. Once this fit is performed, the background is determined for the whole FOV, and we can estimate the background at the source position.

\subsection{Spectral Analysis}

For each flaring source, a time frame was selected by eye to encompass the rise through decline of the flare (see Figure 2). The source extraction region was reduced to a circular region with a radius of $15^{\prime \prime}$ centered on the source. Counts outside the calibrated NUSTAR energy range (3-79 keV) were removed prior to the analysis, and spectra were binned so that each bin included a minimum of 30 counts. The high end of the energy range for the spectral analysis was further limited by low statistics (i.e., not enough counts at higher energies to make a bin with at least 30 counts), and most spectra extend up to $\sim 20 \mathrm{keV}$. The spectral analysis was performed for each source in XSPEC (version 12.9.0u), simultaneously fitting data from both FPMA and FPMB (see Figure 3). EL 29 falls close to the chip gap for FPMA (closer than for FPMB) during this observation, which is likely the cause of the difference in normalization between the focal plane modules for this spectrum.

\subsubsection{Single Temperature Model}

For each source, the flare data were modeled as an optically thin thermal plasma (vapec) with an absorption component (tbabs) to account for attenuation by circumstellar material, which is mainly important at lower X-ray energies. The free parameters for this model, labeled " $1-\mathrm{T}+\mathrm{abs}$ " in Table 2, included temperature $(k T)$, absorption column $\left(N_{\mathrm{H}}\right)$, and a normalization factor $(n)$. From this normalization, we compute an emission measure (EM) by using the
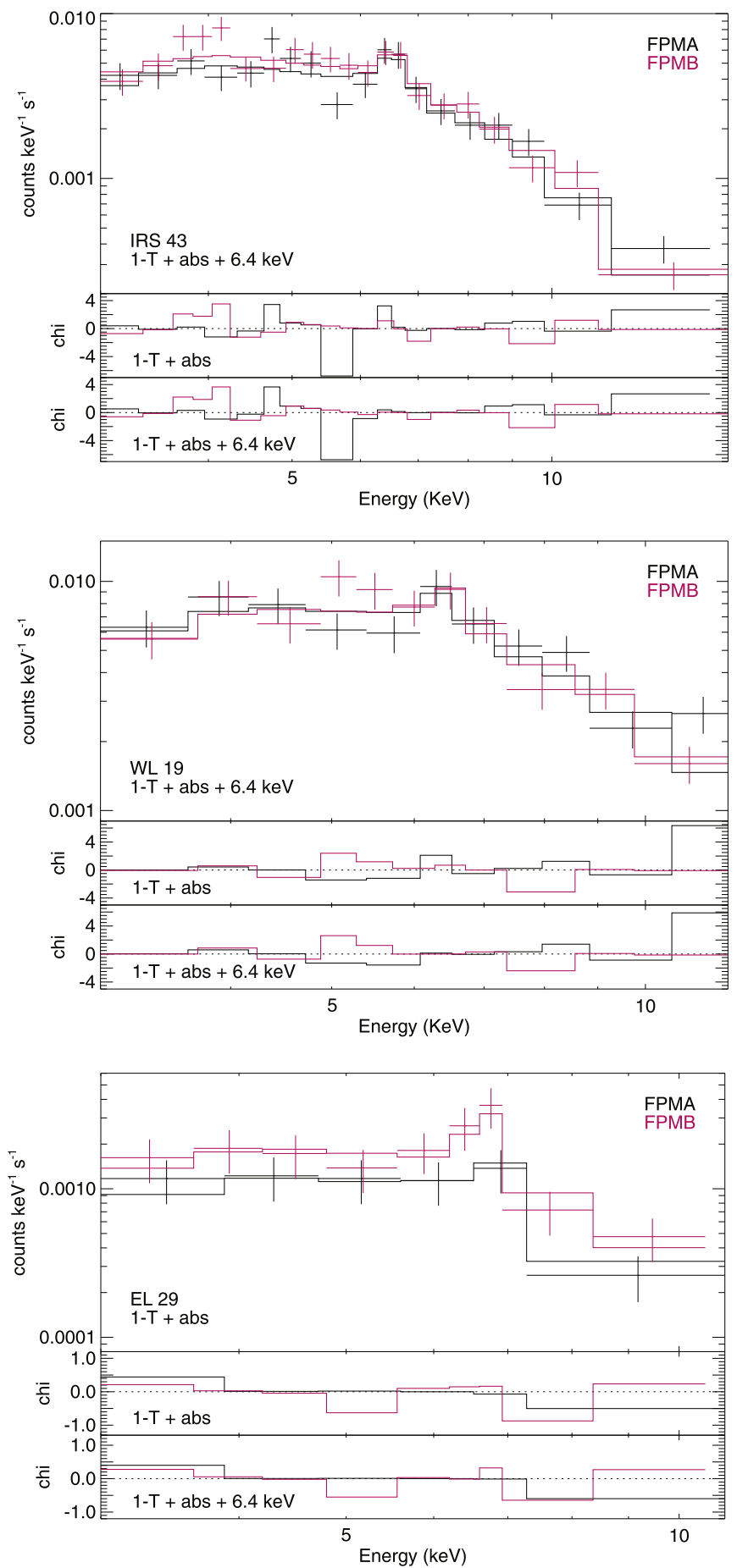

Figure 3. Flare spectra for (top) IRS 43, (middle) WL 19, and (bottom) EL 29. The top panel of each plot shows data from FPMA (black) and FPMB (magenta) along with the best-fit model. Data from FPMA and FPMB are simultaneously fit, with all parameters tied together except for a cross-normalization factor. EL 29 falls close to the chip gap for FPMA (closer than for FPMB) during this observation, which is likely the cause of the difference in normalization between the focal plane modules for this spectrum. The middle panel shows the contribution to the chi-squared value, with sign according to the difference of the data and the model for each data point using an optically thin thermal plasma model (vapec) plus an absorption component (tbabs). With this model, excess emission is observed around $6.4 \mathrm{keV}$ for IRS 43 and WL 19 (but not for EL 29). The bottom panel shows the contribution to the chi-squared value for each data point when a $6.4 \mathrm{keV}$ line is added to the model. For the flares in IRS 43 and WL 19, including this additional emission line improves the fit. 
Table 2

Flare Model Parameters from the Spectral Analysis Described in Section 3.2

\begin{tabular}{|c|c|c|}
\hline Parameters & $1-\mathrm{T}+\mathrm{abs}$ & $1-\mathrm{T}+\mathrm{abs}+6.4 \mathrm{keV}$ \\
\hline \multicolumn{3}{|c|}{ IRS 43} \\
\hline$N_{\mathrm{H}}^{\mathrm{a}}\left(10^{22} \mathrm{~cm}^{-2}\right)$ & $2.3_{0.0}^{4.9}$ & $2.3^{\mathrm{b}}$ \\
\hline$T^{\mathrm{c}}(\mathrm{MK})$ & $57_{50}^{68}$ & $58_{53}^{65}$ \\
\hline$Z_{\mathrm{Fe}}{ }^{\mathrm{d}}$ & $0.2_{0.1}^{0.4}$ & $0.2_{0.0}^{0.3}$ \\
\hline$n_{A}^{\mathrm{e}}\left(10^{-3} \mathrm{~cm}^{-5}\right)$ & $7.7_{5.9}^{9.8}$ & $7.5_{6.6}^{8.5}$ \\
\hline$\chi_{\text {red }}^{2}$ & 1.2 & 1.1 \\
\hline $\mathbf{E M}^{\mathrm{f}}\left(10^{54} \mathrm{~cm}^{-3}\right)$ & $1.3_{1.0}^{1.7}$ & $1.3_{1.1}^{1.5}$ \\
\hline $\mathbf{E} \mathbf{W}^{\mathrm{g}}(\mathbf{e V})$ & $\cdots$ & 190 \\
\hline \multicolumn{3}{|c|}{ WL 19} \\
\hline$N_{\mathrm{H}}\left(10^{22} \mathrm{~cm}^{-2}\right)$ & $3.4_{0.0}^{7.7}$ & $3.4^{\mathrm{b}}$ \\
\hline$T(\mathrm{MK})$ & $76_{57}^{113}$ & $81_{65}^{102}$ \\
\hline$Z_{\mathrm{Fe}}$ & $0.2_{0.0}^{0.5}$ & $0.1_{0.0}^{0.3}$ \\
\hline$n_{A}\left(10^{-3} \mathrm{~cm}^{-5}\right)$ & $9.8_{6.7}^{14.5}$ & $9.4_{7.9}^{11.3}$ \\
\hline$\chi_{\text {red }}^{2}$ & 1.4 & 1.3 \\
\hline EM $\left(10^{54} \mathrm{~cm}^{-3}\right)$ & $1.7_{1.2}^{2.5}$ & $1.6_{1.4}^{1.9}$ \\
\hline $\mathbf{E W}(\mathbf{e V})$ & $\cdots$ & 230 \\
\hline \multicolumn{3}{|c|}{ EL 29} \\
\hline$N_{\mathrm{H}}\left(10^{22} \mathrm{~cm}^{-2}\right)$ & $7.6^{\mathrm{h}}$ & $7.6^{\mathrm{h}}$ \\
\hline$T(\mathrm{MK})$ & $37_{28}^{52}$ & $38_{28}^{54}$ \\
\hline$Z_{\mathrm{Fe}}$ & $0.6_{0.2}^{1.0}$ & $0.5_{0.1}^{1.0}$ \\
\hline$n_{B}^{\mathrm{i}}\left(10^{-3} \mathrm{~cm}^{-5}\right)$ & $4.3_{2.7}^{6.6}$ & $4.2_{2.5}^{6.4}$ \\
\hline$\chi_{\text {red }}^{2}$ & 0.6 & 0.6 \\
\hline EM $\left(10^{54} \mathrm{~cm}^{-3}\right)$ & $0.7_{0.5}^{1.1}$ & $0.7_{0.4}^{1.1}$ \\
\hline EW (eV) & $\ldots$ & 120 \\
\hline
\end{tabular}

Notes. Subscripts and superscripts on the parameter values indicate the lower and upper limits to the $90 \%$ confidence interval, respectively. Bolded values are those derived from fit parameters.

${ }^{\mathrm{a}} N_{\mathrm{H}}$ : hydrogen column density.

${ }^{\text {b }} N_{\mathrm{H}}$ : fixed to fit value from $1 \mathrm{~T}+$ abs model.

${ }^{\mathrm{c}} T$ : temperature.

${ }^{\mathrm{d}} Z_{\mathrm{Fe}}$ : iron abundance relative to solar (Anders \& Grevesse 1989).

e $n_{A}$ : normalization for FPMA data.

${ }^{\mathrm{f}} \mathrm{EM}$ : emission measure, the distance to the source is $\sim 120 \mathrm{pc}$.

${ }^{\mathrm{g}} \mathrm{EW}$ : equivalent width of $6.4 \mathrm{keV}$ emission line.

${ }^{\text {h }} N_{\mathrm{H}}$ : fixed using best-fit value from Imanishi et al. (2001).

${ }^{\mathrm{i}} n_{B}$ : normalization for FPMB data, used instead of $n_{A}$ due to the chip gap issue noted in Section 3.2.

normalization formula for vapec described in the XSPEC manual $^{5}$ and accounting for the distance to the source-in this case, $\sim 120$ pc (Loinard et al. 2008).

In addition, the vapec model allows for a number of elemental abundances to vary with respect to solar abundances, which provides greater flexibility for working with the differing compositions of young stars. In our model, all lighter elements were fixed to solar abundances (Anders \& Grevesse 1989) while the abundance for iron $\left(Z_{\mathrm{Fe}}\right)$, which is an element affecting our energy range and that is typically less abundant for younger stars (Maggio et al. 2007), was allowed to vary. Data from FPMA and FPMB were simultaneously fit, with all parameters tied between data sets apart from a cross-normalization factor. Fit parameters are shown in Table 2 and spectra are shown in Figure 3.

\footnotetext{
5 https://heasarc.gsfc.nasa.gov/xanadu/xspec/manual/node133. html\#vapec
}

\subsection{2. $6.4 \mathrm{keV}$ Emission Line}

After applying an optically thin thermal plasma model, which already includes an iron emission line at $\sim 6.7 \mathrm{keV}$ from the thermal plasma, the flare spectra for IRS 43 and WL 19 showed excess emission around $6.4 \mathrm{keV}$ (see Figure 3). To account for this excess, we added a Gaussian emission line centered at $6.4 \mathrm{keV}$ with $\sigma=0.1 \mathrm{keV}$ (both fixed) and a normalization parameter that was left free. We note that the flux in the $6.4 \mathrm{keV}$ line will, in a sense, trade off with the iron abundance in the vapec model since our coarse energy binning and $N U S T A R$ 's finite resolution will allow the 6.4 and the 6.7 keV lines to share flux in the bins around 6-7 keV.

Since $N_{\mathrm{H}}$ is not well-constrained in our energy range, we fix this parameter to the value from the initial fit (IRS 43 and WL 19) or to a value found in previous studies of the same sources (EL 29); this reduction of free parameters allows for the normalization of the $6.4 \mathrm{keV}$ line, and hence the equivalent width (EW), to be constrained. Best values for the $\mathrm{EW}$, computed in XSPEC, are shown in Table 2. Upon initial comparison with the "1T+abs" model, the addition of a $6.4 \mathrm{keV}$ line to the model slightly improves the fit for the flares on IRS 43 and WL 19, while there is no improvement in the fit quality for the flare observed on EL 29. Even so, for all sources studied, the uncertainty on the EW is large enough to be consistent with zero, so though some of the fits are suggestive of a possible line, the line is not statistically significant. We note that, though we did not find evidence for a $6.4 \mathrm{keV}$ line for EL 29, observations of EL 29 by Chandra and XMM-Newton have yielded positive detections of the $6.4 \mathrm{keV}$ line, during both flaring and quiescent times (Favata et al. 2005; Giardino et al. 2007).

\subsection{GOES Class}

These YSO flares are clearly very bright since they can be observed from $120 \mathrm{pc}$ away. A common measure for flare magnitude when classifying solar flares is the X-ray intensity of the flare peak as observed by the Geostationary Observational Environmental Satellite (GOES) series of Earth-orbiting spacecraft, ${ }^{6}$ which is then classified from faintest to brightest as GOES class A, B, C, M, or X (X-class $>10^{-4} \mathrm{~W} \mathrm{~m}^{-2}$ ). From our isothermal fits, we can estimate what the GOES flux $\left(\mathrm{W} \mathrm{m} \mathrm{m}^{-2}\right.$ ) would be if an event of a certain temperature and emission measure were to occur at a distance of 1 au from Earth. For the YSO flares presented here, the equivalent GOES class ranges from $\mathrm{X} 10^{4}-10^{5}$, which is roughly 1000 times the classification of the largest solar flare on record (Kane et al. 2005).

\subsection{Neupert Effect}

The physical processes behind these flares can be further understood by considering the time evolution of the X-ray emission. We examined the flares for evidence of the Neupert effect, which describes a relationship in which the nonthermal (higher-energy) X-ray output traces the rate of input of thermal plasma from the footpoints to the flare loop, over timescales shorter than the loop cooling time (Neupert 1968; Veronig et al. 2002).

In order to study this, the light curve of the WL 19 flare was split into three energy bands, including a low- (3-6 keV),

\footnotetext{
6 www.swpc.noaa.gov/products/goes-X-ray-flux
} 


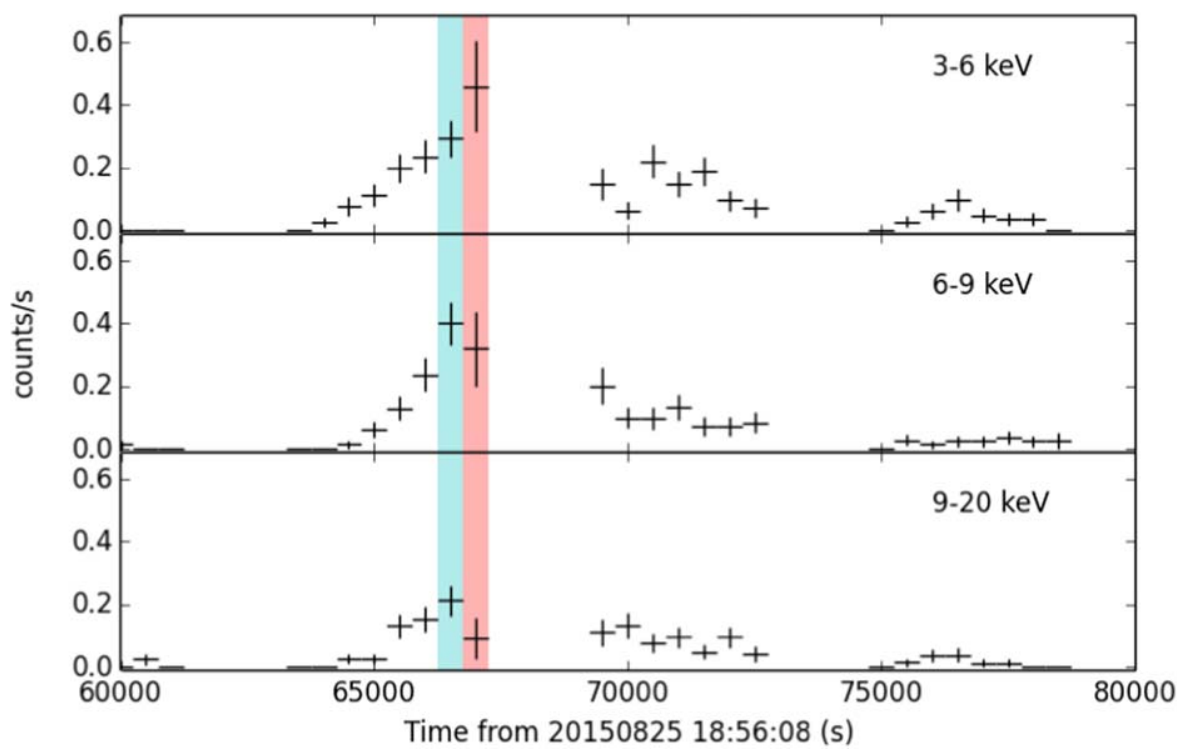

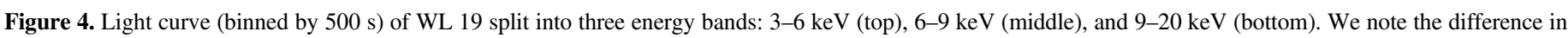

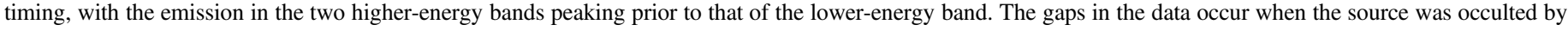
the Earth.

medium- (6-9 keV), and high-energy band (9-20 keV), as seen in Figure 4. From these light curves, we note the difference in peak time between bands, with the two higher-energy bands peaking $\sim 500 \mathrm{~s}$ (one time bin) prior to the lowest energy peak. This effect was not studied in depth for the other two sources due to low statistics and, in the case of IRS 43, gaps in the data because of the source being occulted by the Earth during the rising interval of the flare.

\section{Discussion}

Spectral analysis of three NuSTAR YSO flares, each lasting $15-45 \mathrm{ks}$, found high-temperature plasma ( 40-80 MK) and revealed that these flares are $\sim 1000$ times brighter than the brightest flares on the Sun. An isothermal model effectively described the data for each flare, and no clear evidence for a nonthermal component was found, although the flux at higher energies does peak earlier for the flare on WL 19 (Figure 4). Two of the observed flares, those from IRS 43 and WL 19, showed evidence of an additional emission line at $6.4 \mathrm{keV}$, which is typically attributed to iron fluorescence (discussed further in Section 4.2).

By comparison, our temperatures are found to be consistent with the range of flare temperatures observed in the surveys of $\rho$ Ophiuchi by Chandra (Imanishi et al. 2003) and XMMNewton (Pillitteri et al. 2010). The flares we studied are on the brighter end of those observed in these surveys, which is consistent with our selection method of choosing the brightest events. Similar to our analysis, the Chandra study considered individual flares (in addition to quiescent measurements), and the flare durations are found to be of the same order, averaging $\sim 10-20 \mathrm{ks}$.

\subsection{Flare Energetics}

When considering the energetics of these flares, one thing to address is whether there could be enough energy in nonthermal electrons to account for the observed heating, despite having no clear detection of a nonthermal component above the thermal spectrum. This scenario would allow for a model similar to the standard model for solar flares (Brown 1971) in which energetic electrons deposit energy in the footpoints and heat the ambient plasma, leading to chromospheric evaporation and subsequent thermal emission. The flare on IRS 43 is selected for this energetics analysis since it has the best counting statistics of the sources studied in this paper when integrating over the whole flaring period $(>12 \mathrm{hr})$.

\subsubsection{Nonthermal Electron Energy}

With the flares studied so far, a high-temperature plasma model is sufficient to account for emission at high energies, and we see no clear evidence of nonthermal emission. By assuming that these flares are similar to those observed on the Sun and other stars, scaling laws were used to explore whether or not $N U S T A R$ would be sensitive enough to observe nonthermal emission for flares of the observed magnitudes.

In Battaglia et al. (2005), correlations between parameters for a broad population of solar flares, from GOES class A to class M (soft X-ray flux $\sim 10^{-8}-10^{-4} \mathrm{~W} \mathrm{~m}^{-2}$ ), were studied. By using a scaling relation between the maximum GOES flux and nonthermal flux, we estimated the nonthermal NuSTAR flux at $35 \mathrm{keV}$ to be $\sim 1.4 \times 10^{-6}$ counts $\mathrm{keV}^{-1} \mathrm{~s}^{-1}$ for the flare on IRS 43. In addition, a similar scaling law from Isola et al. (2007) was used to estimate the peak nonthermal NuSTAR flux over the energy range $20-40 \mathrm{keV}$. Along with solar flares, that study also includes a number of stellar flares that are closer in magnitude to the ones observed here by $N u S T A R$. This scaling law estimates the peak nonthermal flux from $20-40 \mathrm{keV}$ to be $\sim 3.5 \times 10^{-5}$ counts $\mathrm{keV}^{-1} \mathrm{~s}^{-1}$. When comparing to the NUSTAR background near this energy range, $\sim 10^{-5}$ counts $\mathrm{keV}^{-1} \mathrm{~s}^{-1}$, we note that the nonthermal flux estimates for IRS 43 are either of the order of or below the NUSTAR background. With the very limited statistics above $20 \mathrm{keV}$ for our observed flares, it is plausible that there could be an undetected nonthermal component among the background. Thus, the absence of a clear nonthermal component to the model does not eliminate the possibility that YSO flares follow the standard model for solar flares. In addition, we are optimistic that NUSTAR may be able to detect a nonthermal 


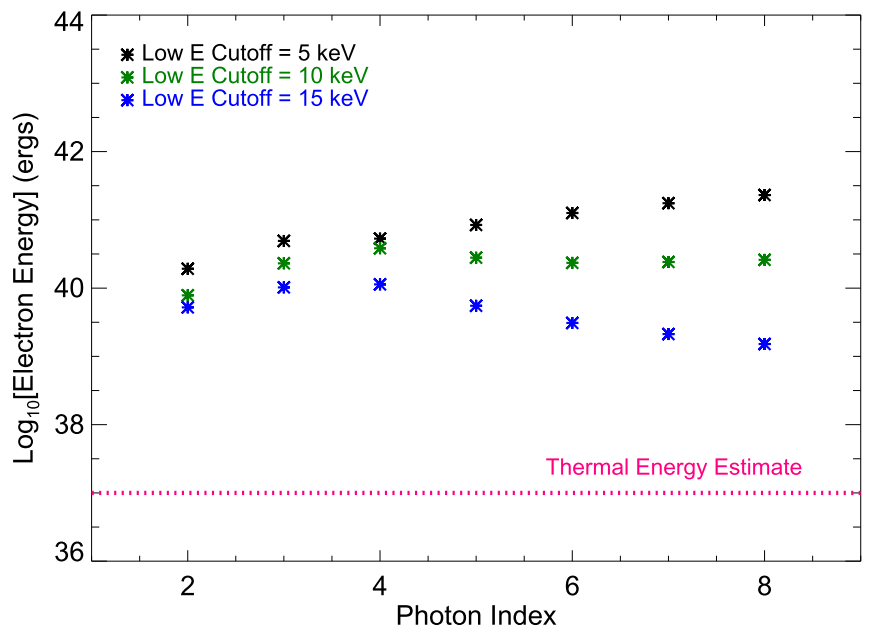

Figure 5. Estimated upper limits on nonthermal electron energy for the flare on IRS 43 over a range of photon indices and cutoff energies of $5 \mathrm{keV}$ (black), $10 \mathrm{keV}$ (green), and $15 \mathrm{keV}$ (blue). For each scenario, the upper limit to the nonthermal electron energy far exceeds the estimated thermal energy (magenta dotted line), indicating that the energy in an undetected nonthermal electron population, if present, could plausibly account for the thermal energy in the flare.

component for larger magnitude flares in future observations of YSOs.

In a separate line of reasoning, we consider how large of a nonthermal component could be present and undetected within our current model. To test this, we add a model to our spectral analysis of IRS 43 that represents nonthermal bremsstrahlung emission-in this case, a broken power-law model (bknpower). Using the thick-target model described in Brown (1971), the parameters from a broken power law in the photon spectrum can be translated into a corresponding electron spectrum with a spectral index, $\delta$, and a low-energy cutoff, $E_{c}$, from which the nonthermal electron energy can be calculated.

For the broken power-law model, the index below an assumed break energy ${ }^{7}\left(E_{\text {break }} \leqslant E_{c}\right)$ is set to $\gamma_{1}=1$, and we test a series of photon indices above $E_{\text {break }}$ at integer intervals from $\gamma_{2}=2$ to $\gamma_{2}=8$. The photon index $\gamma_{2}$ corresponds to the electron spectral index $\delta$ such that $\delta=\gamma_{2}+1$ for a thick-target model. For each $\gamma_{2}$, all parameters of the broken power-law model are fixed, and the normalization is raised until the quality of the fit to the data is affected, ${ }^{8}$ i.e., until the chi-squared value increases by a certain amount. In order to be conservative in our estimate of the plausible nonthermal energy available in the flare, we only allowed for a small increase in chi-squared, ${ }^{9}$ corresponding to a $5 \%$ increase in the confidence with which the fit can be rejected; allowing for additional degradation of the fit quality by raising the normalization further would only increase the amount of nonthermal energy available. With these model parameters, we determine the electron spectrum, test a range of $E_{c}$ values, and compute a rough upper limit on the nonthermal electron energy in the flare (see results in Figure 5). From this plot, we find that the upper limit to the nonthermal

\footnotetext{
$E_{\text {break }}$ is set to $5 \mathrm{keV}$ for $E_{c}=5 \mathrm{keV}$ and set to $10 \mathrm{keV}$ for $E_{c}=10 \mathrm{keV}$ and $E_{c}=15 \mathrm{keV}$.

${ }^{8}$ For other model components (e.g., isothermal plasma, absorption, etc.), the same parameters are left free, as described in Section 3.2.

9 We allowed for an increase in chi-squared from 37.65 to 38.97 (dof $=34$ ), which corresponds to a $5 \%$ increase in the confidence with which the fit can be rejected.
}

electron energy ranges from $\sim 10^{39}-10^{41} \mathrm{erg}$. We note that some combinations of parameters even improved the quality of the fit; the setting an index of $\gamma_{2}=2(\delta=3)$ and a normalization of $\sim 6 \times 10^{-5}$ photons $\mathrm{keV}^{-1} \mathrm{~cm}^{2} \mathrm{~s}^{-1}$ (at $1 \mathrm{keV}$ ) resulted in the best fit of the parameters tested. These parameters correspond to $E_{\mathrm{NT}} \sim(3-4) \times 10^{39} \mathrm{erg}$, depending on the value of $E_{c}$ used.

Given the limited sensitivity of previous X-ray instrumentation above $\sim 10 \mathrm{keV}$, estimates of nonthermal energy for stellar flares in the literature are currently lacking, particularly for YSOs. Studies on surveys of star-forming regions by chargecoupled device (CCD)-based instruments, such as the observations of $\rho$ Ophiuchi by Chandra and XMM-Newton, do not make attempts to place limits on a nonthermal component. A study by Osten et al. (2007), which analyzes a superflare observed by the Swift X-Ray Telescope (XRT) and Burst Alert Telescope (BAT) on the active binary II Pegasi (II Peg), states that it is the first study to provide evidence for a nonthermal component during a stellar flare. Though this is an inherently different flare on a different stellar source, it is nonetheless interesting to note their findings when considering trends for large magnitude events. In this study of II Peg, the best-fit electron spectral indices for two time intervals are $\delta \sim 2.8$ and $\delta \sim 3.1$, which are similar in hardness to the fixed index in our study corresponding to an improved overall fit quality $(\delta=3)$. These findings for extreme stellar events are consistent with patterns observed for solar flares, where a correlation is found between higher nonthermal flux and lower (harder) electron spectral indices (Battaglia et al. 2005). The energetics analysis in Osten et al. (2007) estimates a total electron energy that exceeds the thermal radiative losses; however, challenges with constraining conductive losses in this study make it difficult to state conclusively whether the nonthermal energy can account for total thermal energy in the flare.

\subsubsection{Thermal Energy}

To determine if the estimated upper limits to the nonthermal energy are sufficient to account for the thermal energy, we estimate both radiative and conductive losses for the flare on IRS 43. For an estimate of the radiative losses, we take our thermal model for this flare and extend the model to a broad range of energies in XSPEC. We considered the range of $0.01-200 \mathrm{keV}$, which is a typical energy range for radiation from the corona that has been used in previous stellar X-ray studies (Audard et al. 1999; Osten et al. 2007). By integrating the spectrum over this range, we obtain a radiative flux (erg $\left.\mathrm{cm}^{-2} \mathrm{~s}^{-1}\right)$; the total radiative energy can be computed by accounting for the duration of the flare $(\Delta t)$ and the distance to the source $(D)$. For the observed flare on IRS $43(\Delta t \sim 21 \mathrm{ks}$, $\left.D \sim 3.7 \times 10^{20} \mathrm{~cm}\right)$, the estimated radiated energy released in the corona is $E_{\text {rad }} \sim 6 \times 10^{35} \mathrm{erg}$. We note that this is a lower limit to the radiative losses; multiple studies of solar flares indicate that a majority of the total radiated flare energy comes from the visible and infrared wavebands (Woods et al. 2004, 2006). The actual value for thermal energy could be five times larger than our estimate, bringing the possible value for radiative losses up to $\sim 3 \times 10^{36} \mathrm{erg}$.

In addition to radiative losses, a flare study by Warmuth $\&$ Mann (2016) has also found conductive losses to be significant 
in flares. Conductive energy losses can be computed by

$$
E_{\text {cond }}=\frac{\kappa T^{7 / 2} \mathrm{~A} \Delta t}{l} \mathrm{erg},
$$

where $\kappa$ is the Spitzer conductivity $\left(8.8 \times 10^{-7} \mathrm{erg} \mathrm{cm}^{-1} \mathrm{~s}^{-1}\right.$ $\mathrm{K}^{-7 / 2}$ ), $l$ describes the length scale of energy loss, $A$ is the area of the footpoint, and $\Delta t$ is the flare time interval. For this estimate, we assume a geometry of a cylindrical loop of length $2 l$ with footpoint radius $r$, which is related by the aspect ratio of $\alpha=\frac{r}{2 l}=0.1$ (considered an upper limit for solar flares). With these assumptions incorporated into Equation (1), the conductive losses can be computed in terms of an unknown length, $l$. For the flare on IRS 43 , the conductive energy losses are estimated as $E_{\text {cond }} \sim(4 \times$ $\left.10^{24}\right) \times l$ erg. By freezing the aspect ratio, we note that $A \propto l^{2}$ and thus the conductive losses increase with the loop length rather than being inversely proportional.

We expect that the combined energy in radiative and conductive losses should be equal to the overall energy in the thermal plasma:

$$
E_{\text {therm }}=3 k T \sqrt{E M * V},
$$

where $V$ is the volume of the heated flare plasma. Using this relationship, $E_{\text {therm }} \sim E_{\text {rad }}+E_{\text {cond }}$, and the cylindrical loop geometry described above $\left(V=\pi r^{2}(2 l)\right)$, we can solve for the loop length. Through this process, we estimate $l \sim 5 \times 10^{11} \mathrm{~cm}$, which is consistent with typical values of stellar flare loop lengths ${ }^{10}\left(\sim 10^{11}-10^{12} \mathrm{~cm}\right)$. In a case where conductive losses occur early in the flare, it may be more accurate to assume $E_{\text {therm }} \sim E_{\text {rad }}$. In this scenario, the estimated length is of the same order, with $l \sim 4 \times 10^{11} \mathrm{~cm}$. In either case, the resulting total thermal energy is of the order of $E_{\text {therm }} \sim 10^{37} \mathrm{erg}$, which is orders of magnitude smaller than the upper limits to nonthermal energy, as shown in Figure 5.

Taking this exploration a step further, we can then consider what loop length of $l$ would be required in order for conductive losses to exceed the possible energy in nonthermal electrons $\left(E_{\mathrm{NT}}\right)$. Assuming the upper limit of $E_{\mathrm{NT}} \sim 10^{41} \mathrm{erg}$, an unreasonably large loop length of $l \sim 10^{17} \mathrm{~cm}$ is required for $E_{\text {cond }}>E_{\mathrm{NT}}$. We note that this result assumes a fixed aspect ratio of $\alpha=0.1$, which is considered an upper limit for solar flares; using a smaller aspect ratio would require an even larger loop length for conductive losses to exceed energy in nonthermal electrons. This unlikely scenario further supports the case for the energy in conductive losses being much lower than our upper limits to the nonthermal electron energy. Therefore, we find that electron energy could plausibly account for both radiative and conductive losses in the flare on IRS 43.

\subsection{Iron Fluorescence and Disk Ionization}

By considering the $\mathrm{EW}$ of the $6.4 \mathrm{keV}$ emission line, we can learn more about the ionizing radiation causing fluorescence, the fluorescing material, and the geometry of the source. For IRS 43 and WL 19, we find the best estimates of the EW to be relatively large: $\sim 190 \mathrm{eV}$ and $\sim 230 \mathrm{eV}$, respectively. If we assume that fluorescence comes from photoionization of spherically distributed material around the source, the EW is

\footnotetext{
${ }^{10}$ These values for stellar flare loop lengths were estimated in Shibata \& Yokoyama (1999) using scaling relations and data from a variety of star types, including YSOs, binaries, and red dwarfs.
}

predicted to be around $\sim 10 \mathrm{eV}$, based on $N_{\mathrm{H}}$ and $Z_{\mathrm{Fe}}$ (Inoue 1985). Thus, if we utilize the best-fit EW values for our spectra, the fluorescence cannot be produced by material in the line of sight but instead requires interaction with denser material (George \& Fabian 1991; Sekimoto et al. 1997).

In the case of YSOs, a $6.4 \mathrm{keV}$ line in the flare spectrum with a large $\mathrm{EW}$ is often attributed to fluorescence in the circumstellar disk (Imanishi et al. 2001; Favata et al. 2005; Tsujimoto et al. 2005). Assuming photoionization of disk material, we would expect an EW only up to $120 \mathrm{eV}$, which is still smaller than the best-fit values, aside from that of EL 29 (George \& Fabian 1991). However, in a case where the flare is hidden behind the star's limb, the EW may be larger than $120 \mathrm{eV}$ due to attenuation of the continuum relative to the fluorescence emission from the disk (Drake et al. 2008).

Another way to account for large EWs is through fluorescence by collisional ionization, as was suggested in a study of EL 29 by Giardino et al. (2007). In addition to observing relatively large EWs $(>200 \mathrm{eV})$, this study found that a source of accelerated electrons could better explain the significant variability in EWs than changes in the thermal spectrum.

Given the large uncertainties of the EWs for our study (noted in Section 3.2.2), we refrain from proposing one specific mechanism for our flares but highlight that the potentially large EW may be consistent with a scenario where the disk serves as the main fluorescing material as opposed to the photosphere or material in the line of sight.

One important question regarding high-energy X-ray emission is how this emission impacts the process of planet formation in the protoplanetary disk (Feigelson \& Montmerle 1999; Glassgold et al. 2000; Feigelson 2010). The presence of a $6.4 \mathrm{keV}$ line and the associated EW provide evidence that X-rays from these YSO flares are plausibly interacting with their surrounding disks. X-ray emission can potentially alter the dynamics of the disk by ionizing disk material and, therefore, coupling this material to the magnetic field that, at sufficient ionization levels, could lead to MRI and eventually magnetohydrodynamical turbulence (Balbus 2011).

For a "typical" quiescent YSO $\left(k T=1 \mathrm{keV}, L \sim 10^{29} \mathrm{erg} \mathrm{s}^{-1}\right)$, work by Krolik \& Kallman (1983) estimates that ionization by stellar X-rays could dominate ionization of disk material out to $\sim 1000$ au and that much of the outer disk layer could be sufficiently ionized for MRI. During flaring times, both higher plasma temperatures and higher luminosities can lead to further penetration and an increased ionization rate (Glassgold et al. 2000). For our observed flares, we find $k T$ ranging from $3-7 \mathrm{keV}$ and average luminosities from $10^{30}-10^{31} \mathrm{erg} \mathrm{s}^{-1}$, which would correspond to broader ionization than what is found for the "typical" values. However, work by Ilgner \& Nelson (2006) indicates that the timescale for MRI is much longer than the duration of these X-ray flares, so more research is needed to determine whether transient X-ray events can lead to persistent turbulence in the disk.

\section{Summary}

During three $\sim 50 \mathrm{ks}$ observations of the $\rho$ Ophiuchi cloud complex by $N U S T A R$, multiple bright X-ray flares from YSOs were observed. NuSTAR offers unprecedented sensitivity in the hard X-ray regime above $\sim 7 \mathrm{keV}$, making this data set the first of its kind. Spectral analyses of flares on IRS 43, WL 19, and EL 29 found temperatures ranging from $\sim 40-80 \mathrm{MK}$ and emission measures of the order of $10^{54} \mathrm{~cm}^{-3}$ using an isothermal 
model. These results offer confirmation of what has been found for temperatures and brightnesses in previous X-ray surveys of flaring YSOs in the $\rho$ Ophiuchi region.

The flares presented here show no clear evidence for a higher-energy nonthermal component, but estimates through scaling laws indicate that it may be possible to observe nonthermal emission with a hotter and brighter flare in future observations. Estimates were made of thermal radiative and conductive energy along with upper limits to the energy in nonthermal electrons for the flare on IRS 43. According to our estimates, the energy in nonthermal electrons, if present, could plausibly account for both radiative and conductive losses, which is consistent with the standard model for solar and stellar flares.

Spectral analyses of the flares on IRS 43 and WL 19 suggest the presence of a $6.4 \mathrm{keV}$ emission line. In these cases, the large estimated EW may be consistent with a scenario where flare radiation interacts with dense material in the surrounding disk, producing fluorescence through photoionization. In addition to fluorescence, X-rays from flaring YSOs may significantly ionize disk material, depending on the flare luminosity, temperature, and frequency. Additional modeling and observations in the high-energy X-ray regime with $N U S T A R$ are necessary in order to further investigate the impact of these extreme stellar events.

This work was supported by NSF grants AGS-1429512 and AGS-1752268 and by NASA Headquarters under the NASA Earth and Space Science Fellowship Program Grant 80NSSC17K0430. The authors would like to acknowledge and thank Eric Feigelson for encouraging the NuSTAR team early on to make these observations and for contributing to discussions of this analysis.

Software: NuSTARDAS (v1.6.0), nuskybgd (Wik et al. 2014), XSPEC (Arnaud 1996).

\section{ORCID iDs}

Juliana T. Vievering (1D https://orcid.org/0000-0002-7407-6740 Lindsay Glesener (D) https://orcid.org/0000-0001-7092-2703

Brian W. Grefenstette (iD https://orcid.org/0000-00021984-2932
David M. Smith (10 https://orcid.org/0000-0002-0542-5759

\section{References}

Anders, E., \& Grevesse, N. 1989, GeCoA, 53, 197

Arnaud, K. A. 1996, adass V, 101, 17

Aschwanden, M. J., Holman, G., O’Flannagain, A., et al. 2016, ApJ, 832, 27

Audard, M., Güdel, M., \& Guinan, E. F. 1999, ApJL, 513, L53

Balbus, S. A. 2011, in Physical Processes in Circumstellar Disks around Young Stars, ed. J. V. Garcia (Chicago, IL: Univ. Chicago Press), 232

Battaglia, M., Grigis, P. C., \& Benz, A. O. 2005, A\&A, 439, 737

Brown, J. C. 1971, SoPh, 18, 489

Drake, J. J., Ercolano, B., \& Swartz, D. A. 2008, ApJ, 678, 385

Elias, J. H. 1978, ApJ, 224, 453

Favata, F., Micela, G., Silva, B., Sciortino, S., \& Tsujimoto, M. 2005, A\&A, 433, 1047

Feigelson, E. D. 2010, PNAS, 107, 7153

Feigelson, E. D., \& Montmerle, T. 1999, ARA\&A, 37, 363

George, I. M., \& Fabian, A. C. 1991, MNRAS, 249, 352

Giardino, G., Favata, F., Pillitteri, I., et al. 2007, A\&A, 475, 891

Glassgold, A. E., Feigelson, E. D., \& Montmerle, T. 2000, in Protostars and Planets IV, ed. V. Mannings, A. P. Boss, \& S. S. Russell (Tucson, AZ: Univ. Arizona Press), 429

Harrison, F. A., Craig, W. W., Christensen, F. E., et al. 2013, ApJ, 770, 103 Ilgner, M., \& Nelson, R. P. 2006, A\&A, 455, 731

Imanishi, K., Nakajima, H., Tsujimoto, M., Koyama, K., \& Tsuboi, Y. 2003, PASJ, 55, 653

Imanishi, K., Tsujimoto, M., \& Koyama, K. 2001, ApJ, 563, 361

Inoue, H. 1985, SSRv, 40, 317

Isola, C., Favata, F., Micela, G., \& Hudson, H. S. 2007, A\&A, 472, 261

Kane, S. R., McTiernan, J. M., \& Hurley, K. 2005, A\&A, 433, 1133

Krolik, J. H., \& Kallman, T. R. 1983, ApJ, 267, 610

Lada, C. J., \& Wilking, B. A. 1984, ApJ, 287, 610

Loinard, L., Torres, R. M., Mioduszewski, A. J., \& Rodríguez, L. F. 2008, ApJL, 675, L29

Maggio, A., Flaccomio, E., Favata, F., et al. 2007, ApJ, 660, 1462

Neupert, W. M. 1968, ApJL, 153, L59

Osten, R. A., Drake, S., Tueller, J., et al. 2007, ApJ, 654, 1052

Pillitteri, I., Sciortino, S., Flaccomio, E., et al. 2010, A\&A, 519, A34

Sekimoto, Y., Tatematsu, K., Umemoto, T., et al. 1997, ApJL, 489, L63

Shibata, K., \& Yokoyama, T. 1999, ApJL, 526, L49

Tsujimoto, M., Feigelson, E. D., Grosso, N., et al. 2005, ApJS, 160, 503

Veronig, A., Vršnak, B., Dennis, B. R., et al. 2002, A\&A, 392, 699

Warmuth, A., \& Mann, G. 2016, A\&A, 588, A116

Wik, D. R., Hornstrup, A., Molendi, S., et al. 2014, ApJ, 792, 48

Wilking, B. A., \& Lada, C. J. 1983, ApJ, 274, 698

Wilking, B. A., Lada, C. J., \& Young, E. T. 1989, ApJ, 340, 823

Woods, T. N., Eparvier, F. G., Fontenla, J., et al. 2004, GeoRL, 31, L10802

Woods, T. N., Kopp, G., \& Chamberlin, P. C. 2006, JGRA, 111, A10S14 